\title{
Investigación
}

\section{Prevalencia de parasitosis intestinal, anemia y desnutrición en niños de un resguardo indígena Nasa, Cauca, Colombia, 2015}

\author{
Prevalence of intestinal parasites, anemia and malnutrition among \\ the children of a Nasa indigenous reservation, Cauca-Colombia, 2015
}

\author{
Incidência de parasitose intestinal, anemia e desnutrição em \\ crianças na reserva indígena de Nasa, Cauca-Colômbia, 2015
}

\author{
Luisa María Gaviria, ${ }^{1}$ Duberney Soscue, ${ }^{2}$ Laura Francisca Campo-Polanco, ${ }^{3}$ Jaiberth Cardona-Arias, ${ }^{4}$ Ana Luz Galván-Díaz ${ }^{5}$ \\ Microbióloga y Bioanalista. Universidad de Antioquia. Colombia. Correo electrónico: mjaca462@gmail.com \\ Microbiólogo y Bioanalista. Universidad de Antioquia. Colombia. Correo electrónico: duver1991@hotmail.com \\ MSC Microbiología, Microbióloga y Bioanalista. Universidad de Antioquia. Colombia. Correo electrónico: laura.campolanco@gmail.com \\ MyB, MSc Epidemiología, MSC Economía aplicada. Universidad de Antioquia. Universidad Cooperativa de Colombia. Colombia. Correo \\ electrónico: jaiberthcardona@gmail.com \\ PhD Microbiología y Parasitología, Universidad de Antioquia. Colombia. Correo electrónico: ana.galvan@udea.edu.co
}

Recibido: 11/06/2016. Aprobado: 31/07/2017. Publicado: 05/08/2017

Gaviria LM, Soscue D, Campo-Polanco LF, Cardona-Arias J, Galván-Díaz AL. Prevalencia de parasitosis intestinal, anemia y desnutrición en niños de un resguardo indígena Nasa, Cauca, Colombia, 2015. Rev. Fac. Nac. Salud Pública, 2017; 35(3): 390-399. DOI: 10.17533/udea.rfnsp.v35n3a09

\section{Resumen}

Objetivo: determinar la prevalencia de parasitosis intestinal, anemia y desnutrición en niños de un resguardo indígena Nasa de Caldono, en el departamento del Cauca, y su distribución según variables clínicas, sociodemográficas y de infraestructura sanitaria. Metodología: estudio transversal con fuente de información primaria. La muestra de estudio estuvo formada por 62 niños, a quienes se les hicieron evaluación parasitológica en materia fecal, mediciones antropométricas para evaluar el estado nutricional y determinar la prevalencia de diferentes tipos de desnutrición y medición de hemoglobina para establecer la anemia. La descripción del grupo se realizó con medidas de resumen para la edad y frecuencias para las demás variables, se calculó la prevalencia de los tres eventos (parasitosis, desnutrición, anemia) y se exploró su asociación con variables independientes mediante pruebas de hipótesis. Se usó el programa SPSS 22.0. Resultados: se encontró una prevalencia de parasitosis intestinal de 95,2\%, anemia de $21,0 \%$ y desnutrición crónica de 35,5\%. A pesar de no hallar asociación estadística con las condiciones sociodemográficas y sanitarias, se encontró elevada frecuencia de factores de riesgo para los tres eventos, como la baja escolaridad de los padres, baja disponibilidad de acueducto y alcantarillado, y una elevada morbilidad sentida. Conclusión: la comunidad indígena evaluada presentó altas prevalencias de parasitosis intestinal, anemia y desnutrición, lo que representa implicaciones prácticas para la orientación de los programas de salud indígena; la exploración de asociaciones requiere estudios con mayor tamaño de muestra que garanticen una mayor potencia estadística.

--------Palabras clave: parasitosis intestinal, anemia, desnutrición, comunidad indígena, salud indígena. 


\begin{abstract}
Objective: to determine the prevalence of intestinal parasitosis, anemia and malnutrition among children of a Nasa indigenous reservation from Caldono in the Colombian department of Cauca, and their distribution according to clinical, sociodemographic and healthcareinfrastructure variables. Methodology: a crosssectional study with a primary source of information. Sixtytwo children were evaluated for intestinal parasites via stool analysis. Similarly, anthropometric measurements were used to assess nutritional status and determine the prevalence of various types of malnutrition. Likewise, the presence of anemia was determined by measuring hemoglobin levels. The group was described using summary measures for age and frequency measures for the other variables. Prevalence was calculated for intestinal parasites, anemia and malnutrition, and its association with independent variables was explored using hypothesis testing. The program SPSS 22.0 was used in this study. Results:
\end{abstract}

The prevalence values were: $95.2 \%$ for intestinal parasites, $21 \%$ for anemia and $35.5 \%$ for chronic malnutrition. Although there was no statistical association with sociodemographic and health conditions in the study group, a high frequency of risk factors for intestinal parasites, anemia and malnutrition was found. These factors were: parents with low schooling levels, low availability of aqueducts and sewerage and high perceived morbidity. Conclusion: The evaluated indigenous community had a high prevalence of intestinal parasites, anemia and malnutrition. This has practical implications for the direction that healthcare programs targeting indigenous populations should take. Exploring the associations requires further studies with larger sample sizes which guarantee greater statistical power.

--Key words: intestinal parasites, anemia, malnutrition, indigenous community, indigenous health.

\section{Resumo}

Objetivo: determinar a incidência de parasitose intestinal, anemia e desnutrição em crianças da reserva indígena de Nasa de Caldono, no Departamento de Cauca, e sua distribuição segundo variáveis clínicas, sociodemográficas e de infraestrutura sanitária. Metodologia: Estudo transversal com fonte de informação primária. A mostra de estudo esteve formada por 62 crianças, nas quais foram realizadas avaliações parasitológicas em material fecal, medições antropométricas para que se fosse avaliado o estado nutricional e determinada a incidência de diferentes tipos de desnutrição, e medição de hemoglobina para que se determinasse a anemia. A descrição do grupo realizou-se com medidas de síntese por idade e frequências; para as demais variáveis, calculou-se a incidência dos três eventos (parasitoses, desnutrição, anemia), e explorouse sua associação com as variáveis independentes mediante verificação da hipótese. Utilizou-se o programa SPSS 22.0.
Resultados: verificou-se uma incidência de parasitose intestinal de $95,2 \%$, anemia de $21,0 \%$, e desnutrição crônica de $35,5 \%$. Apesar de não ter sido comprovada uma associação estatística entre as condições sociodemográficas e sanitárias, verificou-se uma elevada frequência de fatores de risco para os três eventos, como a baixa escolaridade dos pais, baixa disponibilidade de água tratada e saneamento, assim como uma elevada morbidade manifestada. Conclusão: a comunidade indígena avaliada apresentou altas incidências de parasitose intestinal, anemia e desnutrição, o que representa implicações práticas para a orientação dos programas de saúde indígena; a exploração de associações requer estudos com maior grandeza de amostra que garantam um maior poder estatístico.

-Palavras-chave: parasitose intestinal, anemia, desnutrição, comunidade indígena, saúde indígena.

\section{Introducción}

Las parasitosis intestinales humanas son infecciones o enfermedades producidas por parásitos cuyo hábitat natural parcial es el aparato digestivo humano. El otro componente del hábitat es el ambiente natural, representado por el suelo, el agua, un animal, etc. Los parásitos intestinales humanos causan trastornos gastrointestinales, hematológicos, nutricionales y de otra índole. Las parasitosis intestinales humanas se consideran un grave problema de salud pública en países de bajos ingresos, especialmente en regiones tropicales como Colombia, en donde las deficientes condiciones socio-económicas e higiénico-sanitarias influyen en la elevada presencia y transmisión de estos parásitos [1].
Para diagnosticar una parasitosis intestinal humana es importante tener en cuenta tres aspectos fundamentales: mecanismo de transmisión, fuente de infección y la presencia de un hospedero susceptible. Dicha transmisión sucede por el consumo de agua o alimentos contaminados con materia fecal, de una persona o animal infectado, por penetración de larvas por piel o consumo de carne con estadios parasitarios [1]. Entre los factores que favorecen el desarrollo de las parasitosis en la población general de humanos se encuentran los inadecuados hábitos higiénicos (incorrecta disposición de excretas, no usar calzado, contaminación en la manipulación de alimentos), convivencia con animales, bajo nivel de escolaridad y la 
insuficiencia de servicios básicos como carencia de agua potable, inadecuada infraestructura sanitaria y problemas de acceso a servicios diagnósticos y terapéuticos [1-3].

Los preescolares, principalmente de hogares pobres, suelen ser los más afectados por este tipo de infecciones, ya que es una población vulnerable y con poco discernimiento de los hábitos adecuados para prevenirlas. Algunas parasitosis intestinales afectan directamente el estado nutricional del menor al generar carencia de hierro, vitamina A y anemia, debido a la afección directa de la mucosa intestinal y sus funciones de absorción y digestión, lo que conlleva la alteración de su estado nutricional, capacidad de aprendizaje y cognición, y daño sobre el estado general de salud del menor [1-3]. Con esto, se evidencia que la desnutrición, la anemia y las parasitosis intestinales presentan convergencias clínicas, a lo que se suman su nexo epidemiológico al compartir factores de riesgo sociodemográficos y de infraestructura sanitaria, lo que constituye un problema de salud pública que requiere especial atención y eficiente intervención.

Por otra parte, las comunidades indígenas constituyen un grupo con una alta vulnerabilidad económica y de salud, debido a la confluencia de múltiples factores como la pobreza, la carencia de servicios básicos y el escaso acceso a servicios de salud, lo que ubica a esta población en una preocupante situación de riesgo de adquirir enfermedades e infecciones. Además, esta población se ha adaptado a vivir en ambientes tropicales, cerca de los ríos, a caminar descalzos para laborar en la agricultura y otras conductas que la hacen vulnerable a adquirir infecciones parasitarias [4].

Por otra parte, según la Encuesta Nacional de la Situación Nutricional en Colombia 2010, uno de cada cuatro niños, entre 6 meses y 5 años de edad, sufre de anemia, en mayor proporción en las zonas rurales. En indígenas, la presencia de retraso en el crecimiento fue de $29,5 \%$ y entre los niños el 7,5\% presentó desnutrición global, ambas cifras constituyen más del doble de la frecuencia reportada en población no indígena [5]. Si bien la Encuesta Nacional de Parasitismo Intestinal en escolares no explicita resultados para los diferentes grupos étnicos, se presentó una mayor frecuencia en zonas con asentamientos indígenas, siendo más alta la prevalencia global en la Sierra Nevada de Santa Marta, con 74,1\%, y en la Amazonía, con 81,6\% [6].

Particularmente, en lo referido a antecedentes de investigación en estos temas, en comunidades indígenas colombianas se dispone de estudios que evidencian una elevada prevalencia de parasitosis intestinales humanas, diversidad en los tamaños de muestra y el principal grupo poblacional evaluado correspondiente a niños. En estos antecedentes se encontraron prevalencias de parasitosis intestinal entre $73 \%$ y $96,4 \%$, predominando los siguientes parásitos: Ascaris lumbricoides, Entamoeba histolytica/dispar, Giardia intestinalis, Trichuris trichiura, Endolimax nana, Entamoeba coli, Iodamoeba butschlii, Blastocystis spp., Entamoeba hartamanni y Uncinarias [7-11]. Es de resaltar que dichos antecedentes resultan limitados en la identificación de otros agentes parasitarios, en la medida en que solo incluyen la tamización con el método directo y un método de concentración, lo que redunda en el sub-registro de la prevalencia de protozoos como Cryptosporidium spp., el cual es uno de los principales parásitos asociados con diarreas en población infantil y requiere de tinciones especiales para su identificación [12].

En estudios previos, realizados en una comunidad indígena ubicada en el departamento de Caldas (Colombia), se encontró que, de los niños que presentaron desnutrición crónica (retraso en la talla para la edad), 8\% presentó anemia [11]. Otros estudios han evidenciado la elevada frecuencia de parasitismo intestinal $\mathrm{y}$ desnutrición en el mismo tipo de población. Así, en niños indígenas de Tierralta (Colombia) se reportó una prevalencia de desnutrición crónica moderada y grave de $63,6 \%$, y parasitosis intestinal en $78,1 \%$ de los niños evaluados [7]. Estos antecedentes de investigación en Colombia indican que, en conjunto, la anemia, la desnutrición y las parasitosis intestinales pueden afectar el mismo tipo de población y agudizar los problemas de salud del menor que las presente.

La comunidad indígena Nasa se ubica principalmente en el departamento del Cauca y en menor proporción en el Valle del Cauca, Putumayo, Tolima, Huila, Caquetá y Meta. La mayor densidad poblacional se encuentra en los municipios de Toribío, Páez y Caldono. Este pueblo tiene características que favorecen la presencia de parásitos intestinales, entre las que se encuentra la de residir en zonas rurales con poco acceso a servicios públicos y de salud [13].

De acuerdo a lo anterior, y teniendo en cuenta la poca información relacionada con la prevalencia de parásitos intestinales y su asociación con variables nutricionales, hematológicas y sociodemográficas en comunidades indígenas en Colombia, y particularmente del departamento del Cauca, se realizó un estudio con el objetivo de determinar la prevalencia de parasitosis intestinal, anemia y desnutrición en niños de un resguardo indígena Nasa de Caldono, en el departamento del Cauca, y su distribución según variables clínicas, sociodemográficas y de infraestructura sanitaria. Esta investigación genera información relevante para la orientación de planes preventivos y de atención en salud; además, se hace explícita la necesidad de incrementar el número de estudios relacionados con los problemas de salud de las comunidades indígenas. 


\section{Metodología}

\section{Tipo y población de estudio}

Se realizó un estudio descriptivo transversal en 62 niños, entre 1 y 5 años, pertenecientes al Resguardo San Lorenzo, de Caldono, en el departamento del Cauca. Se excluyeron niños cuyos padres de familia no firmaron el consentimiento informado, niños de los que no fue posible obtener información clínica y/o sociodemográfica, y aquellos sin muestra para examen coprológico. Se realizó un muestreo no probabilístico por conveniencia. El tamaño de la muestra corresponde al total de registros obtenidos, luego de que las autoridades del comité de salud indígena convocaran a la comunidad para los días en que asistirían los investigadores al resguardo para la recolección de la información.

Área de estudio: el Resguardo San Lorenzo, de Caldono, se encuentra ubicado en la zona Andina, en la vertiente occidental de la cordillera central, a los $2^{\circ}$ $48^{\prime \prime}$ y $3^{\circ} 19^{\prime \prime}$ Latitud norte $-76^{\circ} 05^{\prime}$ y $76^{\circ} 50^{\prime \prime}$ Longitud oeste, y en el sector oriental del departamento del Cauca. Su área es de $373.98 \mathrm{Km}^{2}$. Limita por el Este con los municipios de Jambaló y Silvia; al Oeste con los municipios de Morales y Piendamó; al Sur con los municipios de Silvia y Piendamó; y al Norte con los municipios de Santander de Quilichao y Buenos Aires. El número de habitantes es de 8.679 [14], siendo uno de los más grandes y representativos del pueblo Nasa, lo que justificó su elección para esta investigación.

La economía de la etnia Nasa se basa en el policultivo a pequeña escala mediante el uso de tecnología rudimentaria. La etnia tiene la tierra como el principal medio de trabajo y el cultivo de maíz como eje de su economía. La estructura política incluye el Consejo Regional Indígena del Cauca (CRIC) como máxima autoridad, y los Cabildos como autoridades que rigen el destino social y jurídico al interior del resguardo, mediante el Gobernador como líder, seguido por el comisario, el alcalde, el alguacil, el fiscal y finalmente el capitán. La máxima autoridad en lo referido a la salud son los médicos tradicionales "The Walas" [15].

\section{Recolección de la información}

Encuesta sociodemográfica: se aplicó una encuesta clínico-epidemiológica a los acudientes o padres que otorgaron información del niño $\mathrm{y}$ de aspectos socioeconómicos, clínicos e higiénico-sanitarios del hogar. Los componentes evaluados en la encuesta fueron: sexo y edad del niño, escolaridad de los padres o persona a cargo del niño, infraestructura sanitaria (alcantarillado, conexión de agua intra-domiciliaria, basureros y quebradas cerca de la vivienda), eliminación de excretas, convivencia con animales en la vivienda y morbilidad sentida el último mes.
Examen de materia fecal: la muestra de materia fecal de cada niño fue recolectada en frascos con tapa rosca estériles. Para la determinación de parásitos intestinales se analizó cada muestra mediante el método de coprológico directo y concentración formol-éter. Adicionalmente, se realizó la tinción de Ziehl Neelsen modificado para la búsqueda de Cryptosporidium spp. [16]. Las muestras se procesaron en la sección de parasitología de la Escuela de Microbiología de la Universidad de Antioquia, un día después de su recolección en el Resguardo.

Vale precisar que los diagnósticos de Cystoisospora belli y Cyclospora cayetanensis se pueden hacer mediante coprológico directo y por concentración, teniendo en cuenta que presentan ooquistes con un tamaño de 8 a 10 $\mu \mathrm{m}$ (Cyclospora) y 23-36 $\mu \mathrm{m}$ x 12-17 $\mu \mathrm{m}$ (Cystoisospora) $\mathrm{y}$, por lo tanto, pueden ser observados en montajes en solución salina y lugol, con un objetivo de 40X. La tinción Ziehl Neelsen modificada se puede utilizar como una prueba complementaria, como se realizó en este estudio, para mejorar la captación de casos. En el caso de Cryptosporidium, teniendo en cuenta su tamaño (3-6 $\mu \mathrm{m})$, fue necesaria la tinción para realizar una adecuada búsqueda de los ooquistes. En cuanto al método de concentración utilizado (Ritchie modificado/formol éter), es de sedimentación útil, tanto en la concentración de protozoos como de helmintos; para estos últimos se prefieren los métodos de flotación, teniendo en cuenta el tamaño de los huevos de estos parásitos [17-20].

Determinación de la concentración de hemoglobina: la concentración de hemoglobina fue determinada mediante el método de cianometahemoglobina en un fotómetro portable (Hemocue $\mathrm{Hb} 21+$ ), usando controles y concentraciones estándares. Los puntos de corte para anemia fueron: para niños entre 1 y 4 años de edad $<11,0$ $\mathrm{g} / \mathrm{dL}$ y niños mayores de 5 años $<11,5 \mathrm{~g} / \mathrm{dL}$; sin embargo, los valores de hemoglobina fueron ajustados de acuerdo con la altitud del resguardo indígena $(-0,5 \mathrm{~g} / \mathrm{dL}$ para una altura sobre el nivel del mar entre 1.500 y $1.999 \mathrm{~m}$ ) [21].

Medidas antropométricas: utilizando balanzas y tallímetros debidamente calibrados, se evaluó el estado nutricional de la población infantil, empleando el programa Anthro Plus (para niños de 0 a 5 años) de la OMS, en los que se generan tres indicadores: peso para la edad $(\mathrm{P} / \mathrm{E})$ para evaluar la desnutrición global, talla para la edad (T/E) en desnutrición crónica e índice de masa corporal para la desnutrición aguda. Con el índice de masa corporal para la edad se clasificó en desnutrición en el caso de valores inferiores a -2 desviaciones estándar, y en riesgo de desarrollarla cuando se hallaran valores menores a -1 desviación estándar.

\section{Análisis estadísticos}

Para describir las variables del estudio, se usaron medidas de resumen y frecuencias. Se calculó la 
prevalencia de parasitismo intestinal y de infección por parásitos comensales, patógenos, protozoos, helmintos y por especie identificada. Se determinó la prevalencia de anemia y malnutrición aguda, crónica y global. Para explorar la asociación de los tres eventos con las condiciones sociodemográficas y sanitarias del grupo de estudio, se comparó la frecuencia de parasitismo intestinal, anemia y desnutrición con la escolaridad de los padres a través de prueba chi cuadrado de tendencia lineal (para datos ordinales), con las características de infraestructura sanitaria, convivencia con animales y morbilidad sentida con la prueba chi cuadrado de Pearson (para datos nominales), y con el grupo de edad (categorizada en 1-3 años y 4-5 años) y el sexo con la prueba exacta de Fisher (para variables dicotómicas y frecuencia esperada menor de 5). Se determinó la fuerza de la asociación con razones de prevalencia y su intervalo de confianza del 95\%. Los análisis se realizaron en SPSS 22.0 y Epidat 3.0, con una significación del 0,05.

\section{Aspectos éticos}

El proyecto fue avalado por el comité de Bioética de la Universidad Cooperativa de Colombia (Informe de aprobación: 0800-009) y las autoridades del Resguardo.
Se tuvieron en cuenta los principios de la Resolución 8430 de 1993, que clasifica este estudio como riesgo mínimo y de la Declaración de Helsinki de la Asociación Médica Mundial. Se firmó consentimiento informado de los padres de familia de cada niño, en el cual se informó objetivo, riesgos, beneficios del estudio, así como el carácter confidencial y voluntario de la participación en el estudio. A todos los participantes se les entregó un paquete alimentario y a los que obtuvieron muestras positivas para parásitos se les entregó el medicamento antiparasitario con la respectiva fórmula médica.

\section{Resultados}

En los niños estudiados, la edad media fue $3,3 \pm 1,1$ con rango de 1-5 y rango intercuartil 2-4. Se halló una mayor proporción de hombres y en la morbilidad sentida la mayor frecuencia fue en enfermedad diarreica y dolor abdominal. En las variables de los padres se observó un $60 \%$ con primaria incompleta y $23 \%$ con primaria completa. En la descripción de las variables de la vivienda se halló un $42 \%$ sin alcantarillado, $84 \%$ sin conexión a agua intra-domiciliaria, $71 \%$ con eliminación de excretas en pozo séptico y alta proporción de convivencia con animales (Tabla 1).

Tabla 1. Características sociodemográficas de los niños de un resguardo indígena Nasa de Caldono, Departamento del Cauca

\begin{tabular}{|c|c|c|c|}
\hline & Variable & Número & Porcentaje \\
\hline \multirow{2}{*}{ Sexo } & Mujer & 28 & 45,2 \\
\hline & Hombre & 34 & 54,8 \\
\hline \multirow{5}{*}{ Edad (años) } & 1 & 1 & 1,6 \\
\hline & 2 & 22 & 35,5 \\
\hline & 3 & 8 & 12,9 \\
\hline & 4 & 22 & 35,5 \\
\hline & 5 & 9 & 14,5 \\
\hline \multirow{3}{*}{$\begin{array}{l}\text { Escolaridad de } \\
\text { persona a cargo }\end{array}$} & Primaria incompleta & 37 & 59,7 \\
\hline & Primaria completa & 14 & 22,6 \\
\hline & Secundaria completa & 11 & 17,7 \\
\hline \multirow{4}{*}{$\begin{array}{l}\text { Infraestructura } \\
\text { sanitaria }\end{array}$} & Alcantarillado & 36 & 58,1 \\
\hline & Conexión agua intra-domiciliaria & 10 & 16,1 \\
\hline & $\begin{array}{l}\text { Recolección de basuras cerca } \\
\text { de la vivienda }\end{array}$ & 37 & 59,7 \\
\hline & Quebradas cerca de la vivienda & 18 & 29,0 \\
\hline \multirow{3}{*}{$\begin{array}{l}\text { Eliminación de } \\
\text { excretas }\end{array}$} & Pozo séptico & 44 & 71,0 \\
\hline & Deposición en campo abierto & 43 & 69,4 \\
\hline & Letrina & 12 & 19,4 \\
\hline \multirow{4}{*}{$\begin{array}{l}\text { Presencia de } \\
\text { animales en la } \\
\text { vivienda }\end{array}$} & Perros & 48 & 77,4 \\
\hline & Gallinas & 51 & 82,3 \\
\hline & Caballos & 15 & 24,2 \\
\hline & Cerdos & 8 & 12,9 \\
\hline
\end{tabular}




\begin{tabular}{llcc}
\hline Variable & Número & Porcentaje \\
\hline & Enfermedad diarreica & 29 & 46,8 \\
& Dolor abdominal & 34 & 54,8 \\
& $\begin{array}{l}\text { Enfermedades de la piel } \\
\text { (manchas, vesículas, ampollas, }\end{array}$ & 28 & \\
& úlceras, etc.) & & 45,2 \\
Morbilidad sentida & Prurito anal & 19 & 30,6 \\
el último mes & $\begin{array}{l}\text { Enfermedad neurológica } \\
\text { (problemas del sueño y la }\end{array}$ & 10 & \\
& $\begin{array}{l}\text { memoria, convulsiones, } \\
\text { trastornos del movimiento, etc.) }\end{array}$ & & 16,1 \\
& Prolapso rectal & 3 & 4,8 \\
& Síndrome anémico (cansancio, & 14 & 22,6 \\
\hline & fatiga, palidez, etc.) & & \\
\hline
\end{tabular}

Se halló un 97\% de niños afiliados al régimen subsidiado y uso de calzado en el $63 \%$. En las viviendas, se reportó un $97 \%$ de convivencia con animales, $94 \%$ de consumo de agua hervida, $97 \%$ en lavado de las manos antes de cocinar, $100 \%$ después de ir al baño. Hubo hacinamiento en el $69 \%$ y cocción de carne en el $90 \%$.

La prevalencia general de parasitismo intestinal fue $95,2 \%$. La prevalencia de parásitos patógenos fue
$93,5 \%$ y, por especie, fue mayor para Blastocystis spp. y Entamoeba coli, con $87,1 \%$ y $72,6 \%$, respectivamente; la prevalencia de anemia fue $31 \%$. Se halló una prevalencia de desnutrición crónica del $35,5 \%$, aguda de $8,1 \%$ y global de $13 \%$ (Tabla 2), con problemas de doble carga nutricional en el grupo de estudio, en la medida en que también se halló un $21,0 \%(\mathrm{n}=13)$ de niños con sobrepeso y $3,2 \%(n=2)$ con obesidad.

Tabla 2. Prevalencia de parasitismo, anemia y desnutrición

\begin{tabular}{|c|c|c|c|}
\hline & & Número & Prevalencia \% \\
\hline \multirow{5}{*}{$\begin{array}{l}\text { Parasitismo } \\
\text { intestinal }\end{array}$} & General & 59 & 95,2 \\
\hline & Comensales & 55 & 88,7 \\
\hline & Patógenos & 58 & 93,5 \\
\hline & Protozoos & 58 & 93,5 \\
\hline & Helmintos & 27 & 43,5 \\
\hline \multirow{12}{*}{$\begin{array}{l}\text { Parasitismo } \\
\text { por especie }\end{array}$} & Blastocystis spp. & 54 & 87,1 \\
\hline & Entamoeba coli & 45 & 72,6 \\
\hline & Endolimax nana & 31 & 50,0 \\
\hline & Entamoeba histolytica/dispar/moshkovskii & 29 & 46,8 \\
\hline & Entamoeba hartmanni & 26 & 41,9 \\
\hline & Ascaris lumbricoides & 20 & 32,3 \\
\hline & Giardia intestinalis & 19 & 30,6 \\
\hline & Chilomastix mesnili & 19 & 30,6 \\
\hline & lodamoeba butschlii & 16 & 25,8 \\
\hline & Trichuris trichiura & 11 & 17,7 \\
\hline & Cryptosporidium spp. & 2 & 3,2 \\
\hline & Enterobius vermicularis & 1 & 1,6 \\
\hline \multirow{4}{*}{$\begin{array}{l}\text { Anemia y } \\
\text { desnutrición }\end{array}$} & Anemia & 13 & 21,0 \\
\hline & Desnutrición crónica & 22 & 35,5 \\
\hline & Desnutrición aguda & 5 & 8,1 \\
\hline & Desnutrición global & 8 & 12,9 \\
\hline
\end{tabular}


La anemia no presentó asociación con la desnutrición ni con el parasitismo general o por comensales, patógenos, helmintos o protozoos. De igual forma, la presencia de parásitos patógenos no presentó asociación con la desnutrición. Ninguno de los tres eventos presentó asociación con la escolaridad de los padres. La prevalencia general de parasitismo intestinal, así como los subtipos comensales, patógenos, helmintos o protozoos, no presentaron asociación estadística con las condiciones de infraestructura sanitaria, la convivencia con animales ni la morbilidad sentida.

En la Tabla 3 se presentan las prevalencias específicas de parasitismo intestinal, anemia y desnutrición según edad y sexo, encontrándose asociación para la prevalencia de parásitos comensales y patógenos con el grupo de edad, y de los patógenos con el sexo.

La prevalencia de parásitos comensales fue $20 \%$ mayor en los niños con 4-5 años en comparación con el grupo de entre 1-3 años (Razón de prevalencia 1,20; IC95\% $=1,00-1,44)$; mientras que los patógenos fue $15 \%$ mayor (Razón de prevalencia 1,15 ; IC95\%=1,001,31). La prevalencia de patógenos en las mujeres fue $13 \%$ mayor en comparación con los hombres (Razón de prevalencia 1,13 ; IC95\%= $1,00-1,28)$.

Tabla 3. Prevalencia específica de parasitismo intestinal, anemia y desnutrición según edad y sexo

\begin{tabular}{lcccccc}
\hline & \multicolumn{3}{c}{ Grupo de edad (años) } & \multicolumn{3}{c}{ Sexo } \\
& $\mathbf{1 - 3}$ & $\mathbf{4 - 5}$ & p Fisher & Mujer & Hombre & p Fisher \\
\hline Anemia & 16,1 & 25,8 & 0,349 & 42,9 & 2,9 & $0,000^{*}$ \\
\hline Parasitismo & \multicolumn{7}{c}{. } & & & \\
\hline Global & 90,3 & 100,0 & 0,238 & 100,0 & 91,2 & 0,053 \\
Comensales & 80,6 & 96,8 & $0,036^{*}$ & 96,4 & 82,4 & 0,065 \\
Patógenos & 87,1 & 100,0 & $0,016^{*}$ & 100,0 & 88,2 & $0,025^{*}$ \\
Protozoos & 90,3 & 96,8 & 0,291 & 96,4 & 91,2 & 0,406 \\
Helmintos & 48,4 & 38,7 & 0,442 & 50,0 & 38,2 & 0,443 \\
\hline Desnutrición & & & & & \\
\hline Aguda & 9,7 & 6,5 & 1,00 & 7,1 & 8,8 & 1,00 \\
Crónica & 41,9 & 29,0 & 0,426 & 28,6 & 41,2 & 0,424 \\
global & 19,4 & 6,5 & 0,130 & 14,3 & 11,8 & 0,768 \\
\hline
\end{tabular}

\section{Discusión}

En este estudio, la prevalencia de parasitosis intestinal fue de $95,2 \%$, lo que resulta mayor a lo reportado en la Encuesta Nacional de Parasitismo intestinal de Colombia, donde la mayor ocurrencia se registró en la Sierra Nevada de Santa Marta y en la Amazonía, con 74,05\% y 81,6\%, respectivamente [6]. De igual forma, estos hallazgos muestran que la comunidad Nasa presenta unos índices elevados de este problema, en la medida en que en otras poblaciones indígenas se han reportado prevalencias altas (mayores al 70\%), pero menores al hallazgo de este estudio; con excepción de una investigación sobre parasitismo intestinal en indígenas Wiwa de la Sierra Nevada de Santa Marta, donde se halló una prevalencia del 96,4\% [10-11]. Además, difiere de la prevalencia encontrada en un estudio realizado en una comunidad Nasa ubicada en la ciudad de Cali, posiblemente debido a diferencias en las condiciones sociodemográficas de ambos grupos. Por ejemplo, la comunidad Nasa asentada en Cali se encuentra en zona urbana y periurbana, tienen mayor acceso a servicios de salud, mientras que el grupo asentado en el municipio de Caldono se encuentra ubicado en zona rural y tiene escaso acceso a servicios de salud, lo que ubica a este último en una situación de mayor riesgo para adquirir una infección parasitaria [9].

En relación con la prevalencia de anemia, se debe precisar que la OMS destaca este evento como un problema de salud pública moderado cuando su magnitud no supera el $20 \%$, esto implica que, en los niños de la etnia Nasa, este evento es un aspecto de urgente atención y prevención, ya que se obtuvo un valor del 21\% [22]. También resulta mayor a la prevalencia registrada en otras comunidades indígenas, como en el Resguardo Cañamomo-Lomaprieta (Caldas, Colombia), donde fue del 13\%, con ferropenia del 2\% [11]. Sin embargo, la prevalencia de este estudio fue menor a los datos nacionales dado que, según la Encuesta Nacional de la Situación Nutricional en Colombia 2010, uno de cada cuatro niños, entre 6 meses y 5 años de edad, sufre de anemia, en mayor proporción en las zonas rurales [5]. 
La prevalencia de desnutrición crónica fue de $35,5 \%$, la de desnutrición aguda fue de $8,1 \%$ y la de desnutrición global fue de 12,9\%. Estos datos resultan mayores a las estimaciones nacionales, dado que en la Encuesta Nacional de la Situación Nutricional en Colombia del 2010 se observa que la desnutrición global en comunidades indígenas fue de 7,5\% [5], lo que implica retos para el sistema de salud general de Colombia, así como para las autoridades indígenas, dado que la magnitud hallada evidencia la necesidad de mejorar las acciones para combatir con eficacia este problema de salud pública.

En este sentido, la alta prevalencia de desnutrición crónica observada en los niños Nasa es común en comunidades que viven en precarias condiciones económicas, a consecuencia del proceso de adaptación a las condiciones medioambientales desfavorables y a una alimentación escasa en proteínas. Prueba de ello también se encuentra en el informe Profamilia, el cual refiere que en indígenas la presencia de retraso en el crecimiento fue de 29,5\% y entre los niños el 7,5\% presentó desnutrición global. Ambas cifras constituyen más del doble de la frecuencia reportada en población no indígena del país, como reflejo de profundas desigualdades en salud según la etnia $[5,23]$.

En este estudio, la anemia no presentó asociación estadística con la parasitosis intestinal, lo que puede explicarse en el hecho de que esta relación es más frecuente en infecciones por helmintos $\mathrm{y}$, en menor proporción, por protozoos. Entre los helmintos asociados con anemia se encuentra Trichuris trichiura, el cual se adhiere a la mucosa del intestino grueso, causando edema, inflamación y hemorragias [23, 24], aunque en este estudio su prevalecía fue relativamente baja, en comparación con los otros parásitos reportados. Este hallazgo confluye con otros estudios que no han reportado asociaciones estadísticas entre el parasitismo intestinal y las condiciones nutricionales, síntomas de parasitismo intestinal y condiciones hematológicas [11], aunque también es reflejo de limitaciones en el poder estadístico de las asociaciones, dado el bajo tamaño de algunos subgrupos comparados.

En cuanto a la morbilidad sentida, se tuvieron como principales eventos la enfermedad diarreica aguda y el dolor abdominal, los cuales constituyen síntomas gastrointestinales inespecíficos, ya que no son patognomónicos de una infección parasitaria. Además, es importante tener en cuenta que algunas parasitosis intestinales producen infecciones asintomáticas [7].

Por otra parte, la mayoría de los individuos estudiados defecan en pozos sépticos (71\%) y campo abierto $(69,4 \%)$, condiciones que no son adecuadas frente a las infecciones estudiadas, como lo indican los resultados de algunos estudios, en los que la relación entre la eliminación de excretas en pozos sépticos y la presencia de parasitosis intestinal es estadísticamente significativa [25]. En este orden de ideas, vale recabar que solo el $58,1 \%$ del grupo estudiado dispone de alcantarillado, 16,1\% tiene conexión a agua domiciliaria y más del $70 \%$ convive con animales, lo que resulta diferente en otras comunidades indígenas colombianas, en las cuales se han reportado que $96 \%$ dispone de acueducto domiciliario, $53 \%$ de alcantarillado, $26 \%$ de pozo séptico y $69 \%$ convivencia con animales [11].

$\mathrm{Si}$ bien muchas de las condiciones materiales de vida descritas no presentaron asociación estadística con los eventos de estudio (por limitaciones de poder estadístico), sí muestran una alta vulnerabilidad económica y exposición a riesgos no justificados para padecer parasitismo intestinal $\mathrm{y}$, subsecuentemente, anemia y desnutrición. En este sentido, estudios previos en esta comunidad, y en otros grupos indígenas del Cauca, han recomendado que las investigaciones clínicas, epidemiológicas y de salud pública analicen las relaciones e "interseccionalidad" de las mediciones socio-demográficas y las condiciones materiales de vida, como eje de la acción y planificación pública [26].

$\mathrm{Al}$ desagregar la prevalencia de parásitos comensales y patógenos, se obtuvieron resultados comparables con estudios previos, en los cuales en el grupo de los patógenos predominaron Blastocystis spp., Giardia instestinalis y helmintos como Ascaris lumbricoides y Trichuris trichiura [8]. Con respecto a Cryptosporidium spp., se obtuvo un 3,2\% de prevalencia, siendo este hallazgo de alto significado, ya que este se encuentra vinculado a procesos de diarrea y mala absorción en niños, presentándose más gravedad en aquellos que presenten signos de desnutrición grave, un hallazgo descrito en la población evaluada en este estudio. Además, se debe tener presente que la prevalencia de este protozoo generalmente es subestimada, debido a que en la evaluación parasitológica tradicional no se incluyen las pruebas adecuadas para su tamización y detección. Por consiguiente, se hace necesario realizar más estudios sobre la presencia de dicho parásito en comunidades indígenas, ya que es la primera vez que se describe en este tipo de comunidades, y, a la fecha, solo se ha realizado un estudio adicional, que incluyó técnicas para identificación de Cryptosporidium spp. en asentamientos indígenas [25].

Adicionalmente, se debe resaltar que tanto $G$. intestinalis como Cryptosporidium spp. se encuentran asociados con la contaminación fecal de aguas no tratadas [25], lo que permite relacionar su prevalencia en la comunidad Nasa y la falta de acueducto y tratamiento de aguas en la misma.

Con respecto a la edad, esta fue una variable que se asoció con la presencia de parásitos patógenos, lo que concuerda con Romero y López, quienes afirman que la parasitosis alcanza su máxima prevalencia entre los 2 y 
los 6 años de edad, grupo etario en el cual se encontraron los niños estudiados del Resguardo Indígena Nasa. Lo anterior se puede explicar desde el punto de vista de los hábitos de este grupo, ya que no tienen aún comportamientos de higiene adecuada y pueden tener estrecha relación con otros niños o animales que puedan estar parasitados [27].

El nivel de escolaridad de los padres fue bajo. Esto resulta preocupante, dado que este es un factor de riesgo para desarrollar no solo parasitosis intestinales, sino numerosas enfermedades. Esto se argumenta en limitaciones en sus conocimientos y conductas adecuadas de higiene, salud y autocuidado, lo que aumenta la probabilidad de que los niños, hijos de dichos padres, presenten poliparasitismo [28].

Entre las limitaciones o debilidades del estudio se encuentra el bajo tamaño de muestra, que derivó en una baja potencia estadística en el análisis bivariado. A esto se suma el sesgo temporal de los estudios transversales, el carácter exploratorio de las asociaciones bivariadas y los problemas de validez externa al no realizar un muestreo probabilístico. A pesar de estas limitaciones, esta investigación cristaliza múltiples fortalezas de los estudios descriptivos, como el hecho de estudiar simultáneamente varios eventos de salud y múltiples variables independientes en una población poco conocida, generar hipótesis, orientar el diseño de estudios posteriores y la planificación de servicios de salud [29].

A lo anterior debe sumarse la necesidad de complementar los diseños epidemiológicos con abordajes cualitativos, en todo tipo de trabajo con comunidad indígena que pretenda acciones frente a la enfermedad. En este sentido, es necesario incluir, en este tipo de estudios, elementos que den cuenta de "las maneras de relacionamiento, de producción y de organización social de los territorios aborígenes", más al tener presente que el Consejo Regional Indígena del Cauca (CRIC) ha promovido proyectos de recuperación de la identidad Nasa, fortalecer prácticas tradicionales basadas en "el reconocimiento de la diversidad, la reciprocidad de las acciones y el respeto hacia el territorio" [30].

En conclusión, la prevalencia de parasitosis intestinal encontrada en la población estudiada fue muy elevada, hallando mayor proporción de protozoos que de helmintos. Además, se encontró una elevada prevalencia de desnutrición y de anemia. El indicador antropométrico más afectado fue el de talla para la edad, lo cual es un resultado alarmante, ya que el grupo etario estudiado se encuentra en etapa de crecimiento. Los resultados generados presentan implicaciones prácticas en el mejoramiento de los programas de salud integral indígena para controlar, prevenir y detectar oportunamente las parasitosis intestinales y sus potenciales complicaciones para la salud de la población infantil, y de la comunidad en general.

\section{Agradecimientos}

Al Cabildo Indígena de la comunidad Nasa y a la IPS indígena, por facilitar los antiparasitarios y los paquetes alimenticios para los niños participantes en el estudio; además, por haber autorizado la realización del mismo en su comunidad.

Al Grupo de Parasitología de la Facultad de Medicina de la Universidad de Antioquia, por haber facilitado los equipos y reactivos para la determinación de hemoglobina.

\section{Referencias}

1 Martínez De la Ossa D, Arrieta M, Ampudia A, Fernández M, Hernández S, Hoyos F. et al. Parasitosis intestinal. Ciencia y Salud Virtual 2010; 2(1):122-129.

2 Barón M, Liseti R, Páez MC, Pabón M. Estado nutricional de hierro y parasitosis intestinal en niños de Valencia, Estado Carabobo, Venezuela. An Venez Nutr 2007; 20(1): 5-11.

3 Carmona J. Malaria, desnutrición y parasitosis intestinal en los niños colombianos: interrelaciones. Rev Iatreia 2004; 17(4): 354-369.

4 Organización Mundial de la Salud. Nota descriptiva. La salud de los Pueblos indígenas [Internet] 2007 [Acceso 02 de febrero de 2016]. Disponible en: http://www.who.int $/ \mathrm{hhr} /$ Fact $\% 20$ Sheet $\% 20$ Indigenous $\% 20 \mathrm{Health} \% 20 \mathrm{Nov} \% 202007 \% 20 \mathrm{Final} \% 20 \mathrm{SP}$.pdf

5 Profamilia, Instituto Nacional de Salud, Bienestar familiar, Ministerio de la Protección Social. Encuesta Nacional de la Situación Nutricional en Colombia 2010. Bogotá: Profamilia; 2010.

6 Ministerio de Salud y la Protección Social, Universidad de Antioquia. Encuesta nacional de parasitismo intestinal en población escolar 2012-2014. Medellín: Facultad Nacional Salud Pública, Universidad de Antioquia, Ministerio de Salud y la Protección Social; 2015.

7 Restrepo BN, Restrepo MT, Beltrán JC, Rodríguez M, Ramírez RE. Estado nutricional de niños y niñas indígenas de hasta seis años de edad en el resguardo Embera-Katío, Tierralta, Córdoba, Colombia. Biomédica 2006; 26(4): 517-527.

8 Espinosa DY, Gómez NE, Campo LF, Cardona J, Ríos L. Prevalencia de parasitismo intestinal en la comunidad Seminke del resguardo indígena Wiwa de la Sierra Nevada de Santa Marta, 2014. Archivos de Medicina 2015; 11(2): 1-10.

9 Bermúdez A, Flórez O, Bolaños MV, Medina JJ, SalcedoCifuentes M. Enteroparasitismo, higiene y saneamiento ambiental en menores de seis comunidades indígenas. Cali-Colombia. Rev Salud Pública 2013; 15(1): 1-11.

10 Dib JC, Agudelo LA, Vélez ID. Prevalencia de patologías tropicales y factores de riesgo en la comunidad indígena de bunkwimake, Sierra Nevada de Santa Marta. Duazary 2013; 3(1): 38-44. Disponible en: http://revistas.unimagdalena.edu.co/index. $\mathrm{php} /$ duazary/article/view/605/568

11 Cardona-Arias JA, Rivera-Palomino Y, Carmona-Fonseca J. Salud indígena en el siglo XXI: parásitos intestinales, desnutrición, anemia y condiciones de vida en niños del Resguardo Indígena Cañamomo-Lomaprieta, Caldas-Colombia. MED UIS 2014; 27(2): 29-39.

12 Shoultz DA, de Hostos EL, Choy RK. Addressing Cryptosporidium Infection among Young Children in Low-Income Settings: The 
Crucial Role of New and Existing Drugs for Reducing Morbidity and Mortality. PLoS Negl Trop Dis 2016; 10(1): e0004242.

13 Ministerio de Cultura. Nasa (Páez), la gente del agua [Internet] 2016 [Acceso 30 de abril de 2016]. Disponible en: http://observatorioetnicocecoin.org.co/cecoin/files/Caracterizaci\%C3\%B3n\%20 del $\% 20$ pueblo\%20Nasa.pdf

14 Alcaldía Municipal. Exposición de motivos. Proyecto de acuerdo por medio del cual se adopta el Plan de Desarrollo del Municipio de Caldono para el periodo 2012-2015 [libro en Internet] 2016 [Acceso 15 de marzo de 2016]. Disponible en: http://www.crc. gov.co/files/Descargas/InforCGR/ANEXOS/ANEXO\%203-\%20 Conceptos\%20tecnicos\%20municipiosPDM/plan\%20de $\% 20$ desarrollo\%20caldono.pdf

15 Pachón, X. Los Nasa o la gente Páez [Internet] Bogotá: Instituto Colombiano de Cultura Hispánica; 2016 [Acceso 30 de octubre de 2017]. Disponible en: http://www.lablaa.org/blaavirtual/geografia/geohum $2 /$ nasa $1 . h t m$

16 Instituto Nacional de Salud. Manual de procedimientos de laboratorio para el diagnóstico de los parásitos intestinales del hombre. Serie de Normas Técnicas Número 37. Lima: Instituto Nacional de Salud; 2003. p. 101.

17 Ortega YR, Sterling CR, Gilman RH, Cama VA, Díaz F. Cyclospora Species-A New Protozoan Pathogen of Humans. N Engl J Med 1993; 328(18): 1308-1312.

18 Rao AC, Geetha V, Kudva R, Vidhyalakshmi S, Rupashree S. Histology as a Diagnostic Tool for Intestinal Isosporiasis in Immunocompromised Patients. Asian Pac J Trop Biomed 2012; 2(3): 251-252.

19 Restrepo IC, Mazo LP, Salazar ML, Montoya MN, Botero JH. Evaluación de tres técnicas coproparasitoscópicas para el diagnóstico de geohelmintos intestinales. Iatreia 26(1): 15-24.

20 Botero D. Técnicas de laboratorio en parasitología médica. Parasitosis humanas 5a ed. Medellín: CIB; 2012. p. 679-723.

21 Organización Mundial de la Salud. Concentraciones de hemoglobina para diagnosticar la anemia y evaluar su gravedad. Sistema de Información Nutricional sobre Vitaminas y Minerales [Internet] 2016 [Acceso 20 de mayo de 2016]. Disponible en: http:// www.who.int/vmnis/indicators/haemoglobin_es.pdf
22 Organización Mundial de la Salud. Número de países clasificados en función de la importancia de la anemia para la salud pública. Sistema de información nutricional sobre vitaminas y minerales [Internet] 2016 [Acceso 30 de octubre de 2017]. Disponible en: http://www.who.int/vmnis/database/anaemia/anaemia_data_status_t4/es/

23 UNICEF. Estado Mundial de la Infancia 1998 [Internet] 2016 [Acceso 18 de marzo de 2016]. Disponible en: http://www.unicef. org/spanish/sowc98sp/

24 Durán C. ¿Existe asociación entre anemia, parásitos e inapetencia en una población escolarizada en Bucaramanga? Universidad Industrial de Santander. Escuela de Medicina, Departamento de Pediatría 2009 [Internet] 2016 [Acceso 30 de octubre de 2017]. Disponible en: http://repositorio.uis.edu.co/jspui/bitstream/123456789/10216/2/129623.pdf

25 Mora L, Segura M, Martínez I, Figuera L, Salazar S, Fertmín I, et al. Parasitosis intestinales y factores higiénicos sanitarios asociados en individuos de localidades rurales del estado Sucre. Kasmera 2009; 37(2): 148-156.

26 Urrea F, Rodríguez DA. Transformaciones sociodemográficas y de condiciones de vida en dos pueblos indígenas y poblaciones negras en el norte del Cauca en el período intercensal 1993-2005. Colombia Médica 2012; 43(2): 127-133.

27 Romero J, López MA. Parasitosis intestinales. Protocolos diagnóstico-terapéuticos de Gastroenterología, Hepatología y Nutrición Pediátrica SEGHNP-Asociación Española de Pediatría AEPED [Internet] 2016 [Acceso 20 de marzo de 2016]; [143-149]. Disponible en: https://www.aeped.es/sites/default/files/documentos/parasitosis.pdf

28 Botero J, Castaño A, Montoya MN, Hurtado M, Ocampo NE, Agudelo GM, Cardona OL, et al. Anemia por deficiencia de hierro y su asociación con los parásitos intestinales, en escolares y adolescentes matriculados en instituciones oficiales y privadas de Medellín, 1997-1998. Acta Médica Colombiana 2002; 27(1): 7-14.

29 Cardona JA. Ortodoxia y fisuras en el diseño y ejecución de estudios descriptivos. Revista MED 2015; 23(1): 40-51.

30 Molina VA. Escuela, conocimiento y saber nasa. Aspectos críticos para una política educativa intercultural en Colombia. Actualidades Investigativas en Educación 2015; 15(3): 1-25. 